\title{
Constraints on parameters of B-type pulsators from combined multicolour photometry and radial velocity data
}

\section{I. $\beta$ Cephei stars}

\author{
J. Daszyńska-Daszkiewicz ${ }^{1,2}$, W. A. Dziembowski²,3 and A. A. Pamyatnykh ${ }^{2,4}$ \\ 1 Instytut Astronomiczny, Uniwersytet Wrocławski, ul. Kopernika 11, 51-622 Wrocław, Poland \\ e-mail: daszynska@astro.uni.wroc.pl \\ 2 Copernicus Astronomical Center, Bartycka 18, 00-716 Warsaw, Poland \\ 3 Warsaw University Observatory, Al. Ujazdowskie 4, 00-478 Warsaw, Poland \\ ${ }^{4}$ Institute of Astronomy, Russian Academy of Sciences, Pyatnitskaya Str. 48, 109017 Moscow, Russia
}

Received 13 January 2005 / Accepted 6 June 2005

\begin{abstract}
We analyze data on pulsation amplitudes and phases for two $\beta$ Cephei stars, $\delta$ Cet and $v$ Eri. Strömgren photometry and radial velocity measurements are used simultaneously to obtain constraints on mean parameters of the stars and identification of the excited modes. The inference about the radial mode order and mean star parameters is based on comparison of certain complex parameter, $f$, determined from data, with its counterpart derived from linear nonadiabatic modelling of stellar oscillations. The theoretical $f$ values are very sensitive to the adopted opacity data. In our modelling we rely on the data from OPAL and OP projects. Significant differences were found. New seismic models of $v$ Eri were constructed with both the OPAL and OP opacities.
\end{abstract}

Key words. stars: oscillation (including pulsations) - stars: early-type - stars: atmospheres

\section{Introduction}

In our earlier papers (Daszyńska-Daszkiewicz et al. 2003, 2004) we introduced a new asteroseismic probe of stellar structure. The probe is the complex parameter, $f$, describing the ratio of the relative luminosity variation to the relative radial displacement of the surface. Empirical $f$ values may be derived from pulsation data upon adopting an appropriate stellar atmosphere model. The corresponding theoretical values come from linear nonadiabatic calculations of stellar pulsation. Comparison of the empirical and theoretical $f$ yields a probe of subphotospheric layer. Specifically, this is the layer where the mode period is comparable to the thermal time scale. It means that the layer is not too shallow but still located mostly above the frequency-forming interior. Thus, it is poorly probed by the frequency data.

Our new diagnostic tool was first applied to a sample of $\delta$ Scuti variables (see references above). In that work we were mostly interested in probing the efficiency of subphotospheric convection, whose treatment is a notoriously difficult aspect of stellar interior physics. Calculated $f$ values proved very sensitive to the parametrization of the mixing-length formalism.
In B-stars, treatment of convective transport is not a significant factor in the modelling uncertainty but it is still of interest to ask how well the empirical and theoretical $f$ values agree. The driving effect in B-type pulsators, such as $\beta$ Cephei and SPB stars, arises in the layer of the iron opacity bump. This layer has also the dominant influence on the $f$ parameter. What is essential for the pulsational instability is the location of the layer and the iron abundance. Location depends primarily on the effective temperature. If the overall metallicity parameter, $Z$, describes the iron abundance in the opacity bump layer, then the two crucial parameters, both for the instability and the $f$ value, are $T_{\text {eff }}$ and $Z$. In principle, these two parameters can be derived from spectroscopy. However, there are considerable uncertainties in the derived values which affect accuracy of asteroseismic sounding. We have seen this in the case of the $v$ Eri star (Pamyatnykh et al. 2004; Ausseloos et al. 2004), where the constraints on the convective overshooting from the core were found to be strongly dependent on $T_{\text {eff }}$. In addition, we cannot be sure that the iron abundance in the driving layer is the same as in the photosphere. In fact, Pamyatnykh et al. (2004), facing problems with excitation of certain modes in $v$ Eri, suggested that iron may be accumulated around the opacity bump. Such an effect would surely leave an imprint on the value of $f$. Perhaps such an accumulation could also explain 
the unexpected discovery of a large number of B-type pulsators in Magellanic Clouds (Kołaczkowski et al. 2004).

It is always important to use all available observables to check the accuracy of stellar modelling. A comparison of empirical and theoretical $f$ values provides a stringent test of models of the atmosphere, envelope and pulsation. In addition, the comparison offers an opportunity to test microscopic input physics, especially the opacity data.

Determination of $f$ is possible only in conjunction with identification of the $\ell$ degree for the excited mode. The values of $\ell$ are important for association of measured frequencies with normal modes of stellar oscillations. There are several methods of $\ell$ identifications based on photometry or on spectroscopy, but they required a priori knowledge of $f$ and, frequently, the answer is not unique. In this paper we present the first application of our method of simultaneous determination of $\ell$ and $f$ from combined multi-colour photometry and radial velocity data to $\beta$ Cephei stars.

In the next section we recall our method for simultaneous determination of the $f$ parameter and the $\ell$ degree of an observed mode from multicolour photometry and radial velocity data. Section 3 is devoted to the analysis and interpretation of observational data on $\delta$ Ceti star, which is a monoperiodic $\beta$ Cep variable. The analysis of a multiperiodic $\beta$ Cep star $v$ Eridani is a subject of Sect. 4. A summary and discussion are given in Sect. 5.

\section{The method}

We repeat here the basic formulae underlying our method. The local radial displacement of the surface element is written in the standard form,

$$
\delta r(R, \theta, \varphi)=\operatorname{RRe}\left\{\varepsilon Y_{\ell}^{m} \mathrm{e}^{-\mathrm{i} \omega t}\right\},
$$

where $\varepsilon$ is a small complex parameter fixing mode amplitude and phase, whereas

$Y_{\ell}^{m}(\theta, \phi)=(-1)^{\frac{m+|m|}{2}} \sqrt{\frac{(2 \ell+1)(\ell-|m|) !}{(\ell+|m|) !}} P_{\ell}^{|m|}(\cos \theta) \mathrm{e}^{\mathrm{i} m \phi}$.

With the adopted, non-standard, normalization of spherical harmonics, $|\varepsilon|$ is the rms value of $\delta r / R$ over the star surface. The corresponding changes of the bolometric flux, $\mathcal{F}_{\text {bol }}$, and the local gravity, $g$, are given by

$\frac{\delta \mathcal{F}_{\text {bol }}}{\mathcal{F}_{\text {bol }}}=\operatorname{Re}\left\{\varepsilon f Y_{\ell}^{m} \mathrm{e}^{-\mathrm{i} \omega t}\right\}$

and

$\frac{\delta g}{g}=-\left(2+\frac{\omega^{2} R^{3}}{G M}\right) \frac{\delta r}{R}$

The complex parameter, $f$, which is the central quantity in this work, has been already defined in the Introduction.

Since both $\varepsilon$ and $f$ may be regarded as constant in the atmosphere, we can use the static plane-parallel approximation. Then, the complex amplitude of the relative monochromatic flux variation can be expressed as follows (see e.g. Daszyńska-Daszkiewicz et al. 2002):

$A^{\lambda}(i)=\mathcal{D}_{\ell}^{\lambda}(\tilde{\varepsilon} f)+\mathcal{E}_{\ell}^{\lambda} \tilde{\varepsilon}$ where

$\tilde{\varepsilon} \equiv \varepsilon Y_{\ell}^{m}(i, 0)$,

$\mathcal{D}_{\ell}^{\lambda}=b_{\ell}^{\lambda} \frac{1}{4} \frac{\partial \log \left(\mathcal{F}_{\lambda}\left|b_{\ell}^{\lambda}\right|\right)}{\partial \log T_{\text {eff }}}$,

$\mathcal{E}_{\ell}^{\lambda}=b_{\ell}^{\lambda}\left[(2+\ell)(1-\ell)-\left(\frac{\omega^{2} R^{3}}{G M}+2\right) \frac{\partial \log \left(\mathcal{F}_{\lambda}\left|b_{\ell}^{\lambda}\right|\right)}{\partial \log g}\right]$,

and

$b_{\ell}^{\lambda}=\int_{0}^{1} h_{\lambda}(\mu) \mu P_{\ell}(\mu) \mathrm{d} \mu$.

The $\lambda$ index identifies the passband. The partial derivatives of $\mathcal{F}_{\lambda}\left|b_{\ell}^{\lambda}\right|$ may be calculated numerically from tabular data. In this paper we use data from Kurucz's models (2004). For the limb darkening coefficients we take Claret's fits (2000). To convert the amplitudes to magnitudes, the right hand side of Eq. (1) must be multiplied by the factor $(-1.086)$.

A set of of observational equations for complex unknowns $(\tilde{\varepsilon} f)$ and $\tilde{\varepsilon}$ is obtained from Eqs. (1) written for a number of passbands, $\lambda$. On the left-hand side we have measured amplitudes, $A^{\lambda}$, expressed in the complex form. If we have data on spectral line variations, the set of Eqs. (1) may be supplemented with an expression relating $\tilde{\varepsilon}$ to complex amplitudes of the first moments, $\mathcal{M}_{1}^{\lambda}$,

$\mathcal{M}_{1}^{\lambda}=\mathrm{i} \omega R\left(u_{\ell}^{\lambda}+\frac{G M v_{\ell}^{\lambda}}{R^{3} \omega^{2}}\right) \tilde{\varepsilon}$,

where

$u_{\ell}^{\lambda}=\int_{0}^{1} h_{\lambda}(\mu) \mu^{2} P_{\ell}(\mu) \mathrm{d} \mu$.

$v_{\ell}^{\lambda}=\ell \int_{0}^{1} h_{\lambda}(\mu) \mu\left(P_{\ell-1}(\mu)-\mu P_{\ell}(\mu)\right) \mathrm{d} \mu$.

Another useful quantity from spectroscopy is the complex amplitude of equivalent width variations. This yields an additional constraint on $f$ and $\ell$. We plan to implement this in future work.

The set of Eqs. (1), possibly supplemented with Eq. (2), is solved by the $\chi^{2}$ method for unknown parameters $\tilde{\varepsilon}$ and $(\tilde{\varepsilon} f)$.

Care must be taken in calculating complex observational amplitudes. If the time dependence of the observed quantity, $O$, is given in the form $A_{O} \sin (\omega t+\varphi)$ then the corresponding complex amplitude is $\left(A_{O} \sin \varphi, A_{O} \cos \varphi\right)$. Comparing the empirical and theoretical $f$ values, we have to make sure that in the nonadiabatic pulsation code the time dependence is $\mathrm{e}^{-\mathrm{i} \omega t}$.

Effects of rotation are totally ignored in Eqs. (1) and (2). The approximation is essential because only then unknown values of $i$ and $m$ may be absorbed into $\tilde{\varepsilon}$. As long as the angular velocity of rotation $\Omega$ is much lower than $\omega$ these equations provide a good approximation. Effects of rotation enter the expression for $A^{\lambda}$ through terms $\propto \Omega^{2}$ and the expression for $\mathcal{M}_{1}^{\lambda}$ through terms $\propto \Omega$. Therefore use of Eq. (2) is less safe but, as we will see later, it is essential for mode identification in B-type stars. There is a need for improvement. Fortunately the two stars considered in this paper are slow rotators. 


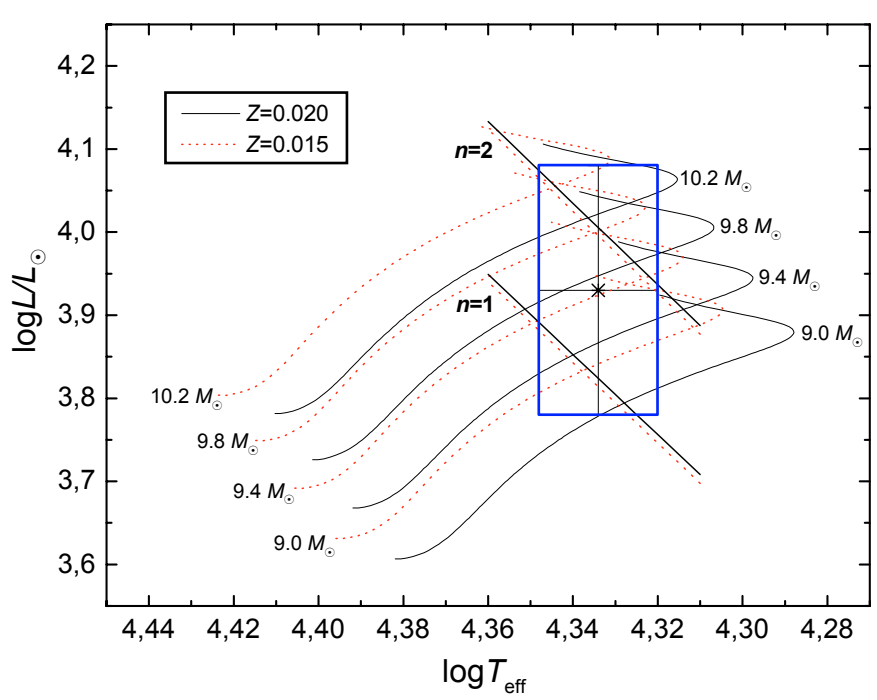

Fig. 1. The observational error box for $\delta$ Ceti in the HR diagram. The evolutionary tracks were calculated for two values of the metallicity parameter $Z$. The lines of constant fundamental $(n=1)$ and the first overtone $(n=2)$ period equal to $0.161137 \mathrm{~d}$ are shown. These lines will be discussed later in this section.

In our applications we take $\omega$ from observations, the stellar radius, $R$, from the measured mean luminosity, $L$, and effective temperature, $T_{\mathrm{eff}}$, while the mass, $M$, is taken from evolutionary tracks.

\section{The monoperiodic $\beta$ Cep star $\delta$ Ceti}

\subsection{Data}

We derive mean parameters of the star relying on Strömgren photometry from the catalogue of Stankov \& Handler (2005) and on the Hipparcos parallax taking into account the Lutz-Kelker correction (Lutz \& Kelker 1973). In Fig. 1 we show position of the star with its error box in the HR diagram. Table 1 presents 5 models which were chosen for analysis; all of them are consistent with the observations. The models correspond to the central value (D1) and to four values close to the edges of the error box (D2-D5), and were fixed at $Z=0.02$.

We use here the amplitudes and phases in four Strömgren passbands from Kubiak \& Seggewiss (1990). These photometric observations were done in 1986 . We derived the corresponding radial velocity data from spectra taken in 1987 (Aerts et al. 1992). The data used in our analysis are given in Table 2.

\subsection{The $\ell$ degree of the excited mode}

The only detected mode in this star has been already identified as $\ell=0$ from spectroscopy by Aerts et al. (1992) and from photometry by Cugier et al. (1994). This identification was confirmed by Cugier \& Nowak (1997), who included observations in the UV range from IUE. However, in the photometric identification, the theoretical $f$ values were adopted. Naturally, extracting both $\ell$ and $f$ from observations sets higher requirements on the data. Photometric data are insufficient.
Table 1. Parameters of selected models of $\delta$ Ceti.

\begin{tabular}{ccccc}
\hline \hline & $M / M_{\odot}$ & $\log T_{\text {eff }}$ & $\log L$ & $\log g$ \\
\hline D1 & 9.70 & 4.3340 & 3.930 & 3.783 \\
D2 & 9.00 & 4.3229 & 3.806 & 3.830 \\
D3 & 9.20 & 4.3459 & 3.790 & 3.947 \\
D4 & 10.20 & 4.3232 & 4.041 & 3.650 \\
D5 & 10.40 & 4.3472 & 4.032 & 3.763 \\
\hline
\end{tabular}

Table 2. Amplitudes and phases in radial velocity and in four Strömgren passbands for monoperiodic $\beta$ Cep star $\delta$ Cet with pulsation frequency $6.205875 \mathrm{c} / \mathrm{d}$.

\begin{tabular}{ccc}
\hline \hline & $A\left[\mathrm{~km} \mathrm{~s}^{-1}\right],[\mathrm{mag}]$ & $\varphi[\mathrm{rad}]$ \\
\hline$V_{\mathrm{rad}}$ & $7.4323(723)$ & $1.6416(78)$ \\
$u$ & $0.0263(9)$ & $2.7758(156)$ \\
$v$ & $0.0143(9)$ & $2.7096(156)$ \\
$b$ & $0.0137(9)$ & $2.7291(156)$ \\
$y$ & $0.0128(9)$ & $2.7057(156)$ \\
\hline
\end{tabular}

It is clear from comparison of the $\chi^{2}(\ell)$ dependence, shown in the two panels of Fig. 2, that radial velocity data are essential for determination of the spherical harmonic degree, $\ell$. We see in the upper plot that without radial velocity data, the discrimination between various even degrees is not possible. The lower plot shows that the mode degree must be $\ell=0$. In addition, the lower luminosity models lead to a lower $\chi^{2}$. In Table 3 we provide our solutions for the $\ell=0$ identification. With this identification $\tilde{\varepsilon}$ is equal to the $\delta R / R$ amplitude.

\subsection{Discrimination between two possible radial modes}

Having determined the degree of the excited mode we still have to consider its possible radial orders. In Fig. 1 we show the theoretical HR diagram with lines corresponding to a period of $0.16114 \mathrm{~d}$ for two different radial mode identifications. Both fundamental and first overtone modes are acceptable. Now, for each value of $Z$ and $n$ we have a one dimensional family of models to consider.

We first check whether the $\chi^{2}$ behaviour may help us to discriminate between the two possible choices of $n$. The result shown in Fig. 3 is rather negative. The difference between the two choices is small, especially in the low temperature range, where we reach the lowest $\chi^{2}$ regardless of the $n$ values. On the same plot we show that the metallicity, $[\mathrm{m} / \mathrm{H}]$, microturbulence, $v_{\mathrm{t}}$, and opacity in the atmosphere models do not matter very much for the $\chi^{2}$ values. The metallicity affects the analysis in two ways. The first is through the evolutionary models, hence the surface gravity, the second is through the $[\mathrm{m} / \mathrm{H}]$ parameter in the atmosphere.

The lesson from the $\chi^{2}$ analysis is that the mode is definitely the radial one and that there is some preference for lower effective temperature and luminosity within the error box. However, the discrimination between metallicities and two radial mode orders was not possible. Much more may be 

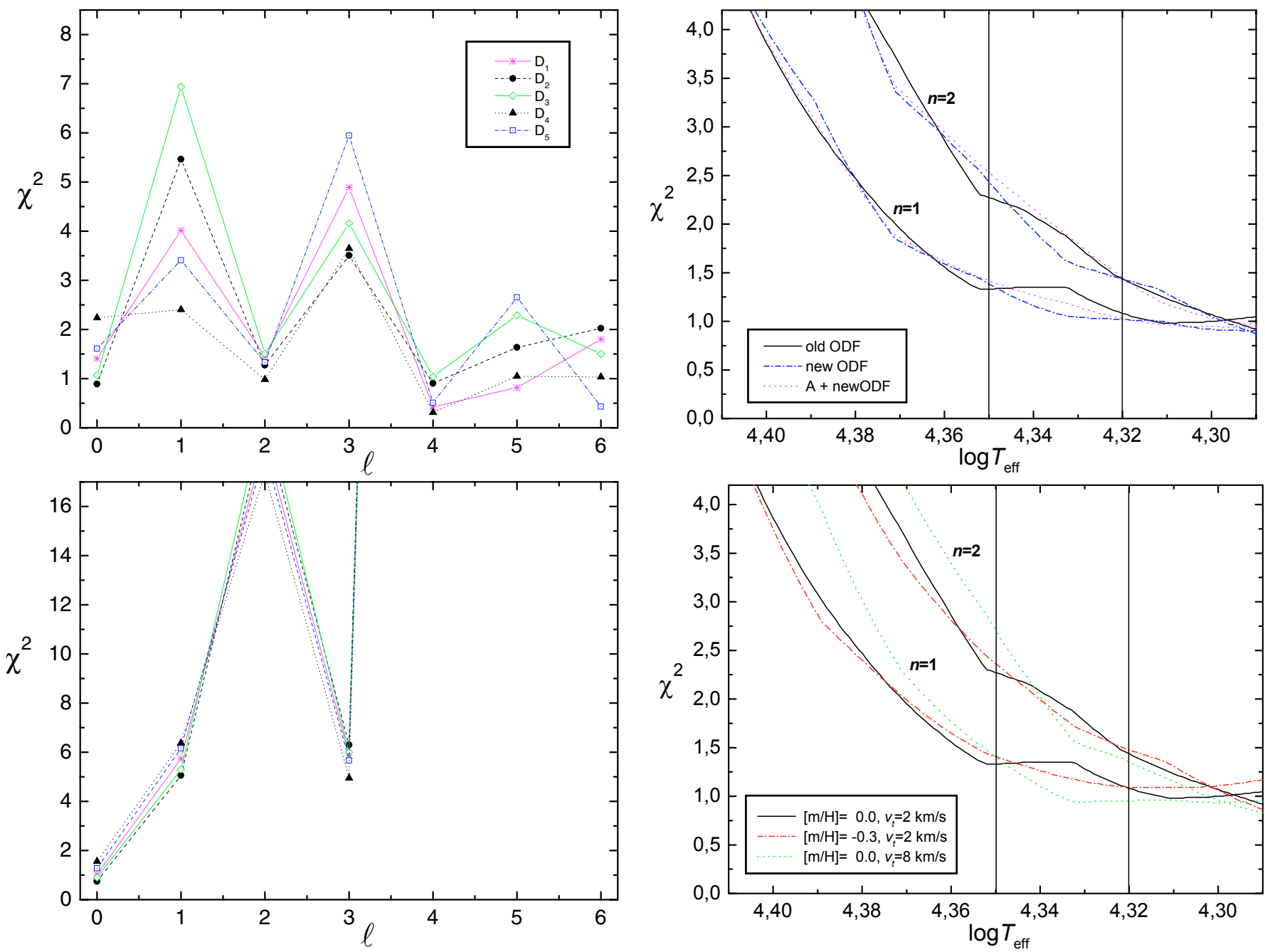

Fig. 2. The $\chi^{2}$ per degree of freedom as a function of $\ell$ obtained from the fit of photometric amplitudes and phases without radial velocity data (upper panel) and with radial velocity data (lower panel). The five lines correspond to models from Table 1 which are consistent with the observations.

Table 3. Values of $\tilde{\varepsilon}, f$ and $\chi^{2}$ for models of $\delta$ Ceti.

\begin{tabular}{cccccc}
\hline \hline & $\tilde{\varepsilon}_{\mathrm{R}}$ & $\tilde{\varepsilon}_{\mathrm{I}}$ & $f_{\mathrm{R}}$ & $f_{\mathrm{I}}$ & $\chi^{2}$ \\
\hline \multicolumn{5}{c}{$v=6.20587 \mathrm{c} / \mathrm{d}, \ell=0$} \\
\hline D1 & $0.00520(5)$ & $0.00036(4)$ & $-9.70(18)$ & $2.56(12)$ & 1.03 \\
D2 & $0.00570(5)$ & $0.00040(4)$ & $-9.13(15)$ & $2.36(10)$ & 0.74 \\
D3 & $0.00645(6)$ & $0.00045(5)$ & $-8.37(14)$ & $2.07(10)$ & 0.90 \\
D4 & $0.00435(5)$ & $0.00031(4)$ & $-1.12(27)$ & $3.05(17)$ & 1.55 \\
D5 & $0.00491(5)$ & $0.00035(4)$ & $-1.01(22)$ & $2.69(14)$ & 1.28 \\
\hline
\end{tabular}

Fig. 3. The $\chi^{2}$ per degree of freedom as a function of $\log T_{\text {eff }}$ for two possible identifications of the radial mode order. The vertical lines show the allowed $T_{\text {eff }}$ range, consistent with the error box shown in Fig. 1. In the upper panel we show the effect of use of the new opacity distribution function (ODF) in the atmosphere and enhancement in $\mathrm{O}$, $\mathrm{Ne}, \mathrm{Mg}, \mathrm{Si}, \mathrm{S}, \mathrm{Ar}, \mathrm{Ca}$ and Ti (A+newODF), following Kurucz (2004). In the lower panel we compare the effect of different metallicity parameters, $[\mathrm{m} / \mathrm{H}]$, and microturbulence velocities, $v_{\mathrm{t}}$.

to the radial mode identification. Comparison of the upper and lower panel strongly suggests that the excited mode is the first overtone. This is the same identification as proposed by Cugier et al. (1994) and supported by Cugier \& Nowak (1997). However there is a problem with this identification, because in the allowed ranges of $T_{\text {eff }}$ and $Z$, the first overtone is stable, as shown in Fig. 5. The adopted measure of instability is $\eta$, which varies between -1 and +1 . For unstable modes we have $\eta>0$.

learnt from comparing the inferred nonadiabatic parameter, $f$, with model calculations.

\subsection{Inference from $f$-values and stability consideration}

As explained in the introduction, the calculated $f$ values must be very sensitive to the iron abundance in the driving zone. The effect is well seen in Fig. 4. We also see the sensitivity gard the discrepancy as serious because in the same range of parameters the fundamental mode is definitely unstable. This is a typical property of $\beta$ Cep star models, in which the lower frequency radial and neighbouring nonradial modes are preferentially unstable.

We may think about various sources of the discrepancy in empirical as well as in theoretical values of $f$. The effect 

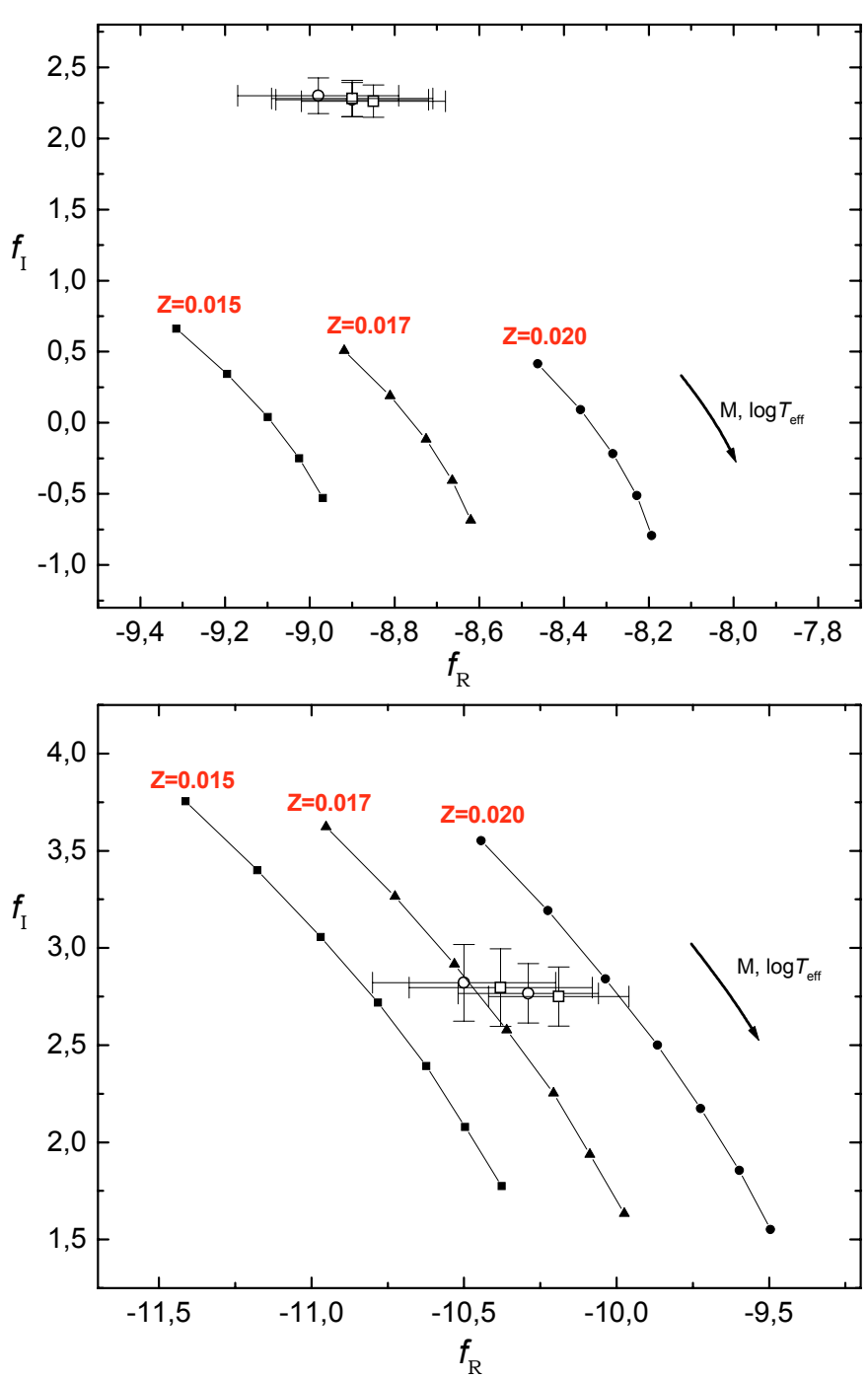

Fig. 4. Comparison of the empirical and theoretical values of $f$ assuming $Z$ and $T_{\text {eff }}$ within the error box. The upper and lower panels refer to the fundamental and first overtone identification of the $\delta$ Cet mode, respectively. The uncertainties in metallicity and effective temperature are included both in the empirical and theoretical values of $f$. The empirical values are taken from Table 3 . The theoretical values were calculated along lines of constant period (see Fig. 1) with the step in mass of $0.2 M_{\odot}$. In the upper panel, the masses at the top are 8.6, 8.8, $9.0 M_{\odot}$ for $Z=0.015,0.017,0.020$, respectively, whereas in the lower panel the masses at the top are 9.2, 9.4, 9.6 $M_{\odot}$ for $Z=0.015,0.017$, 0.020 , respectively.

influencing the latter ones may be related to the opacity data. Fortunately, we have two independent sources of these data. Our results so far were obtained with the tabular opacities from the OPAL project (Iglesias \& Rogers 1996). As the alternative we have latest tables based on the Opacity Project (Seaton \& Badnell 2004; Badnell et al. 2005; Seaton 2005), hereafter OP. As for the relative heavy element abundance, the OPAL data use the GN93 (Grevesse \& Noels 1993) and the OP data use S92 (Seaton et al. 1994) solar mix. The two mixes differ only slightly. In particular, the S92 mix has a Fe abundance higher by $2.5 \%$. Different opacities lead only to small differences in the evolutionary tracks. The OP tracks are fainter

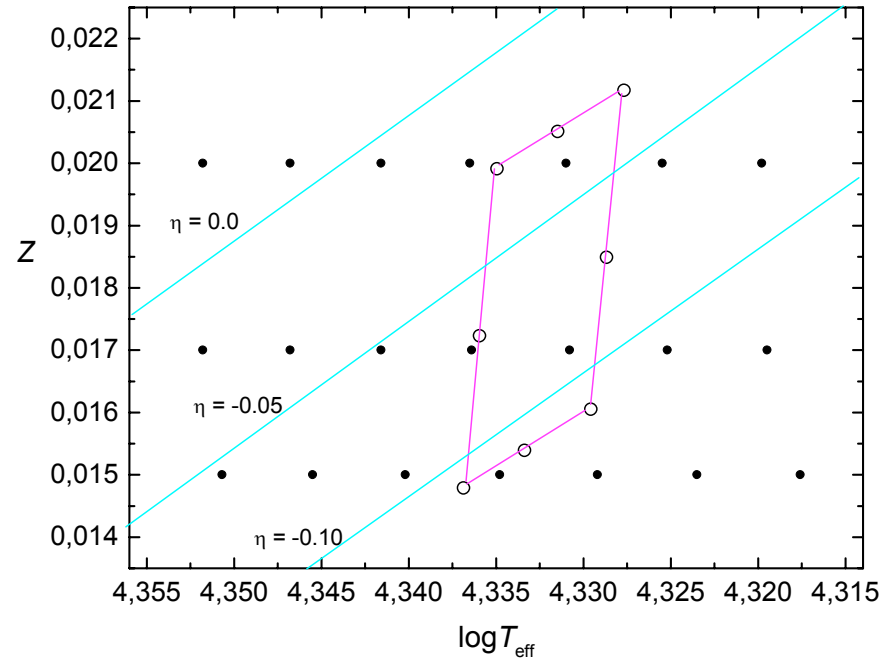

Fig. 5. The ranges of $T_{\text {eff }}$ and $Z$ allowed by consistency of empirical and calculated values of $f$ assuming the first overtone identification. The lines of constant instability parameter, $\eta$, are shown. Negative values of $\eta$ correspond to stability.

by some 0.02 dex. Surprisingly, a large difference was found in the $f$ values. Now, there is no clear discrimination between the fundamental and first overtone based on the comparison of the theoretical and empirical $f$ values. Plots in Fig. 6 show that both identifications are at most marginally within with the error box. Note, however, that while for the fundamental mode identification all acceptable models lie well within the instability domain $(\eta>0)$, the first overtone identification places the models in the stability domain. This is why we prefer the fundamental mode identification, which implies, for instance, that luminosity is lower by 0.33 dex and mass is lower by $2 M_{\odot}$ than in the case of the first overtone identification. Still, improvements are needed for a convincing discrimination between the two possibilities.

\section{The multiperiodic $\beta$ Cep star $v$ Eridani}

This star has been the object of the recent photometric (Handler et al. 2004) and spectroscopic campaigns (Aerts et al. 2004; de Ridder et al. 2004). The number of detected modes is much larger than in any other star of this type. For some of the modes we have unambiguous mode identification, which was supported by seismic model constructions (Pamyatnykh et al. 2004; Ausseloos et al. 2004).

Extracting $f$ values from data for a multiperiodic object is clearly advantageous because we get independent constraints on mean stellar parameters, such as $T_{\text {eff }}$ or $Z$, from each mode.

We adopted the mean parameters of $v$ Eri after Pamyatnykh et al. (2004), where details about derivation are given. In Fig. 7, we show the star position in the HR diagram with the error box. Evolutionary tracks plotted in the figure were calculated for two indicated $Z$ values, assuming no convective overshooting. Seismic models of Pamyatnykh et al. (2004) were obtained at $Z=0.015$. Table 4 presents 5 models which were chosen for analysis, all of them are consistent with the observations. As in the case of $\delta$ Ceti, these five models correspond to the central 

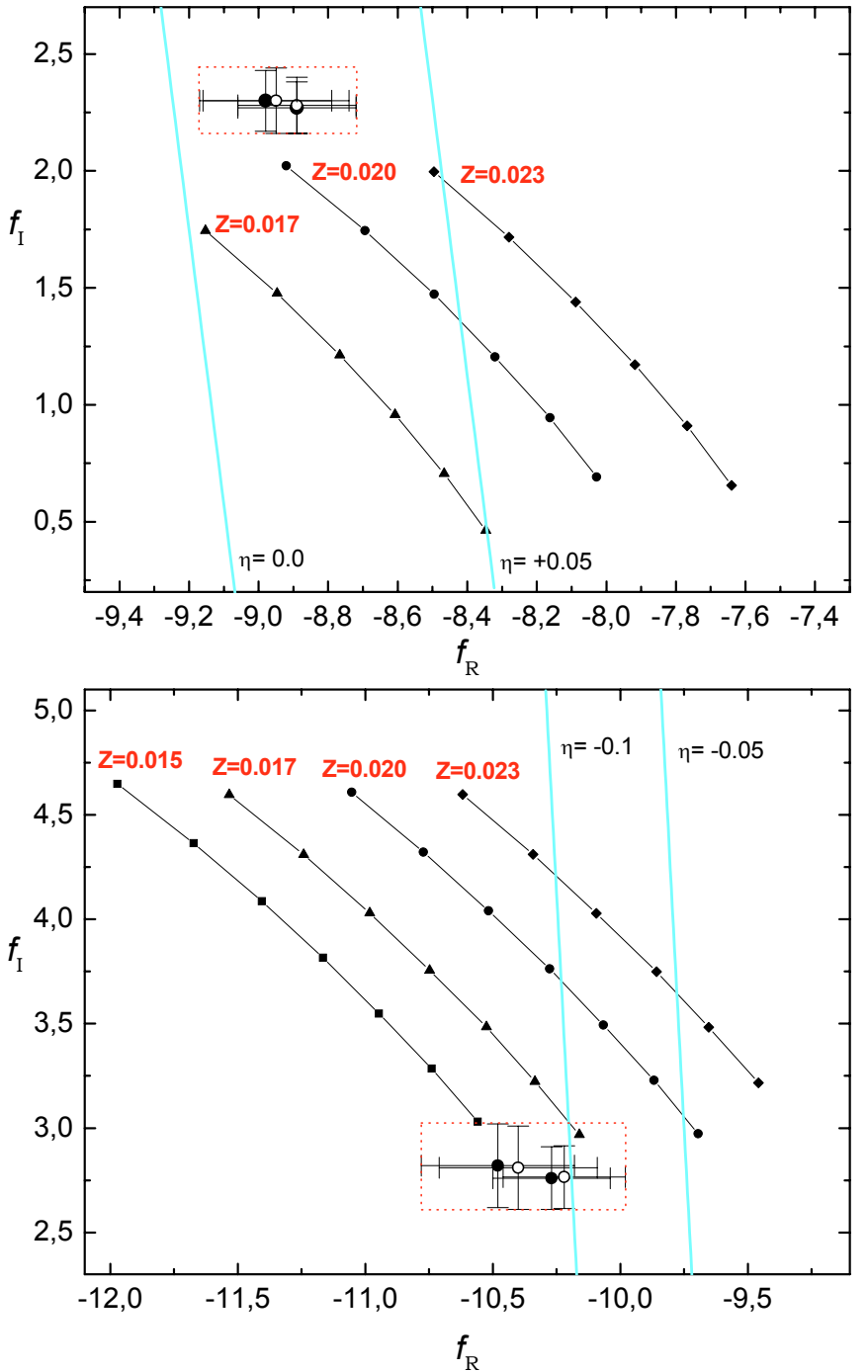

Fig. 6. The same as Fig. 4 but calculated with the new OP opacity tables. In addition, lines of constant values of the instability parameter, $\eta$, are shown. In the upper panel, the masses at the top are $8.8,8.8$, $9.0 M_{\odot}$ for $Z=0.017,0.020,0.023$, respectively, whereas in the lower panel the masses at the top are 9.2, 9.4, 9.6, 9.8 $M_{\odot}$ for $Z=0.015$, $0.017,0.020,0.023$, respectively. The step in mass is $0.2 M_{\odot}$.

value (N1) and to four values close to the edges of the error box (N2-N5), and were fixed at $Z=0.02$.

\subsection{Data}

Photometric amplitudes and phases used in our analysis of this star were kindly provided to us by M. Jerzykiewicz, who reanalyzed data from the multisite observations carried out between October 2002 and February 2003 (Handler et al. 2004). To determine the amplitudes and phases of the radial velocity, we calculated the first moment of the line profile of SiIII $4553 \AA$ using spectra obtained during the last spectroscopic campaign (Aerts et al. 2004). We stress that it is important to use contemporaneous data in this case because amplitudes and phases do change at the level exceeding errors. It is also important that the same frequencies are used in analysis of spectroscopic data. The latter was the reason why we could not rely on results of

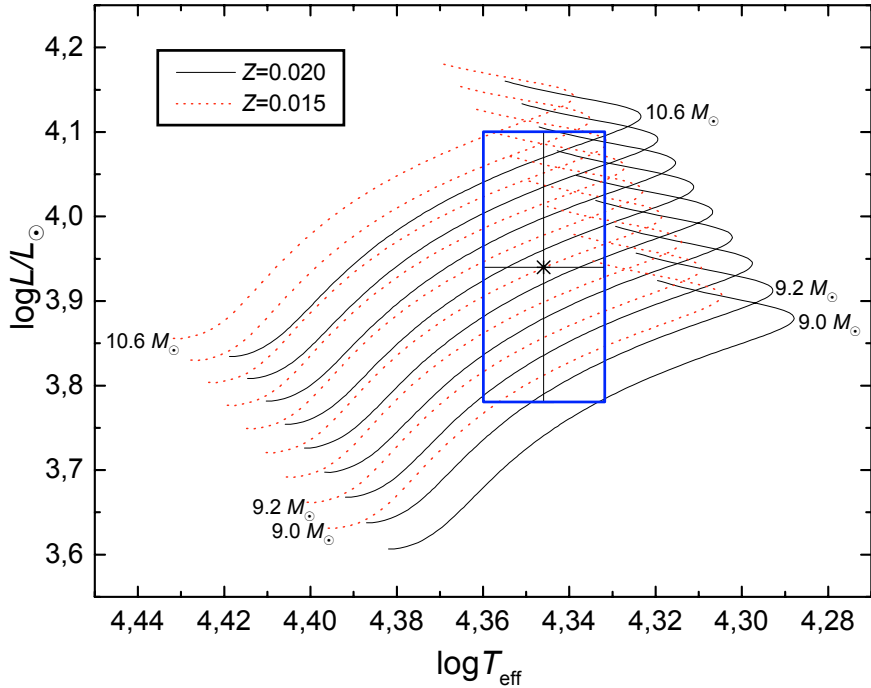

Fig. 7. The observational error box for $v$ Eri on the HR diagram. The evolutionary tracks for two values of the metallicity parameter $Z$ are shown.

Table 4. Parameters of selected models of $v$ Eridani.

\begin{tabular}{ccccc}
\hline \hline & $M / M_{\odot}$ & $\log T_{\text {eff }}$ & $\log L$ & $\log g$ \\
\hline $\mathrm{N} 1$ & 9.90 & 4.3460 & 3.940 & 3.830 \\
$\mathrm{~N} 2$ & 9.20 & 4.3344 & 3.823 & 3.869 \\
$\mathrm{~N} 3$ & 9.40 & 4.3589 & 3.792 & 4.007 \\
$\mathrm{~N} 4$ & 10.40 & 4.3331 & 4.059 & 3.681 \\
$\mathrm{~N} 5$ & 10.60 & 4.3584 & 4.044 & 3.805 \\
\hline
\end{tabular}

De Ridder et al. (2004), who analyzed data from the same campaigns. At the moment, we have sufficiently accurate data only for the four dominant peaks. We have amplitudes and phases in three Strömgren passbands, $u v y$, and the radial velocity data. The data used in our analysis are listed in Table 5.

\subsection{The $\ell$ and $f$ values}

Like in the case of $\delta$ Cet, only after combining photometric and spectroscopic data, could we get a unique $\ell$ identification for the dominant modes in $v$ Eri. Our $\ell$ degrees are the same as those of our predecessors. However, unlike them, we did not assume the $f$ values.

In Fig. 8, we compare $\chi^{2}$ values obtained with and without the radial velocity data for the dominant mode, $v_{1}$, which has been identified as $\ell=0$ (see e.g. De Ridder et al. 2004) on the base of photometric data alone but upon assuming theoretical $f$. We see in the upper part of the figure that our method without spectroscopic data does not allow for $\ell$ determination. In the case of combined data, the minimum of $\chi^{2}$ is indeed very deep, however the value of $\chi^{2}$ is large $(\approx 50)$. Also one gets a very large $\chi^{2}(\approx 40)$ adopting $f$ from theory and relying only on photometric data (De Ridder et al. 2004). For the three modes, $v_{2}, v_{3}, v_{4}$, forming the close triplet, we confirm in Fig. 9 the $\ell=1$ identification. In Table 6 we provide results of our analysis for all four modes but only $\ell$ leading to the $\chi^{2}$ minima. 
Table 5. Amplitudes and phases in radial velocity and in four Strömgren passbands for four dominant frequencies in $v$ Eri.

\begin{tabular}{|c|c|c|}
\hline & $A\left[\mathrm{~km} \mathrm{~s}^{-1}\right],[\mathrm{mag}]$ & $\varphi[\mathrm{rad}]$ \\
\hline \multicolumn{3}{|c|}{$v_{1}=5.76326 \mathrm{c} / \mathrm{d}$} \\
\hline$V_{\mathrm{rad}}$ & $22.017(116)$ & $5.473(5)$ \\
\hline$u$ & $0.07345(20)$ & $0.616(3)$ \\
\hline$v$ & $0.04103(14)$ & $0.590(3)$ \\
\hline$y$ & $0.03685(13)$ & $0.579(4)$ \\
\hline \multicolumn{3}{|c|}{$v_{2}=5.65391 \mathrm{c} / \mathrm{d}$} \\
\hline$V_{\mathrm{rad}}$ & $9.049(115)$ & $0.419(13)$ \\
\hline$u$ & $0.03793(21)$ & $1.982(5)$ \\
\hline$v$ & $0.02645(14)$ & $1.982(6)$ \\
\hline$y$ & $0.02506(13)$ & $1.975(5)$ \\
\hline \multicolumn{3}{|c|}{$v_{3}=5.62009 \mathrm{c} / \mathrm{d}$} \\
\hline$V_{\mathrm{rad}}$ & $8.272(112)$ & $0.129(14)$ \\
\hline$u$ & $0.03464(21)$ & $1.681(6)$ \\
\hline$v$ & $0.02388(15)$ & $1.691(6)$ \\
\hline$y$ & $0.02267(14)$ & $1.684(6)$ \\
\hline \multicolumn{3}{|c|}{$v_{4}=5.63715 \mathrm{c} / \mathrm{d}$} \\
\hline$V_{\mathrm{rad}}$ & $7.703(105)$ & $3.033(14)$ \\
\hline$u$ & $0.03217(22)$ & $4.537(7)$ \\
\hline$v$ & $0.02244(15)$ & $4.537(7)$ \\
\hline$y$ & $0.02105(14)$ & $4.544(7)$ \\
\hline
\end{tabular}

The minima for the triplet are significantly lower than for the $\ell=0$ mode, but still larger than 1 . All these modes have close frequencies thus they should have similar values of $f$. At frequencies around the fundamental radial mode and above, these values are nearly $\ell$-independent, as long as $\ell$ is not large. It is so because for such modes pulsation is vertical in the layers contributing to $f$. Figure 10 shows that indeed all four $f$ values determined from the data are close, which is reassuring. However, the large $\chi^{2}$ are of concern. There are two possible explanations: either the model is inadequate or the errors are underestimated.

Let us begin with the first possibility. Neglect of the effect of rotation in the model is fully justified because the star is a very slowly rotating object. The ratio of rotation to pulsation frequency is $\sim 10^{-3}$. More suspect may seem our use of linear relation between the displacement and the the relative flux perturbation which is only an approximation, but it seems justified. Consider the $v_{1}$ mode, which was identified as radial and for which we determined the highest $\chi^{2}$ (still by far the lowest than with other identification). With such an identification we have $|\varepsilon|=|\tilde{\varepsilon}|=0.012$ and $|\varepsilon f|=0.11$. The maximum relative perturbation temperature is about 0.03 , which is small. The most natural interpretation of the higher order peaks (harmonics and combinations) found e.g. by Handler et al. (2005) is a nonlinearity of the $A^{\lambda}(\varepsilon)$ dependence. The lowest-order nonlinear departure from Eq. (1) is cubic. There is a number of third peaks but there is none at $3 v_{1}$. The peak at $2 v_{1}$ is seen with the amplitude $1 / 16$ of the $v_{1}$ peak. We use these facts as an argument for the applicability of Eq. (1) in our case.
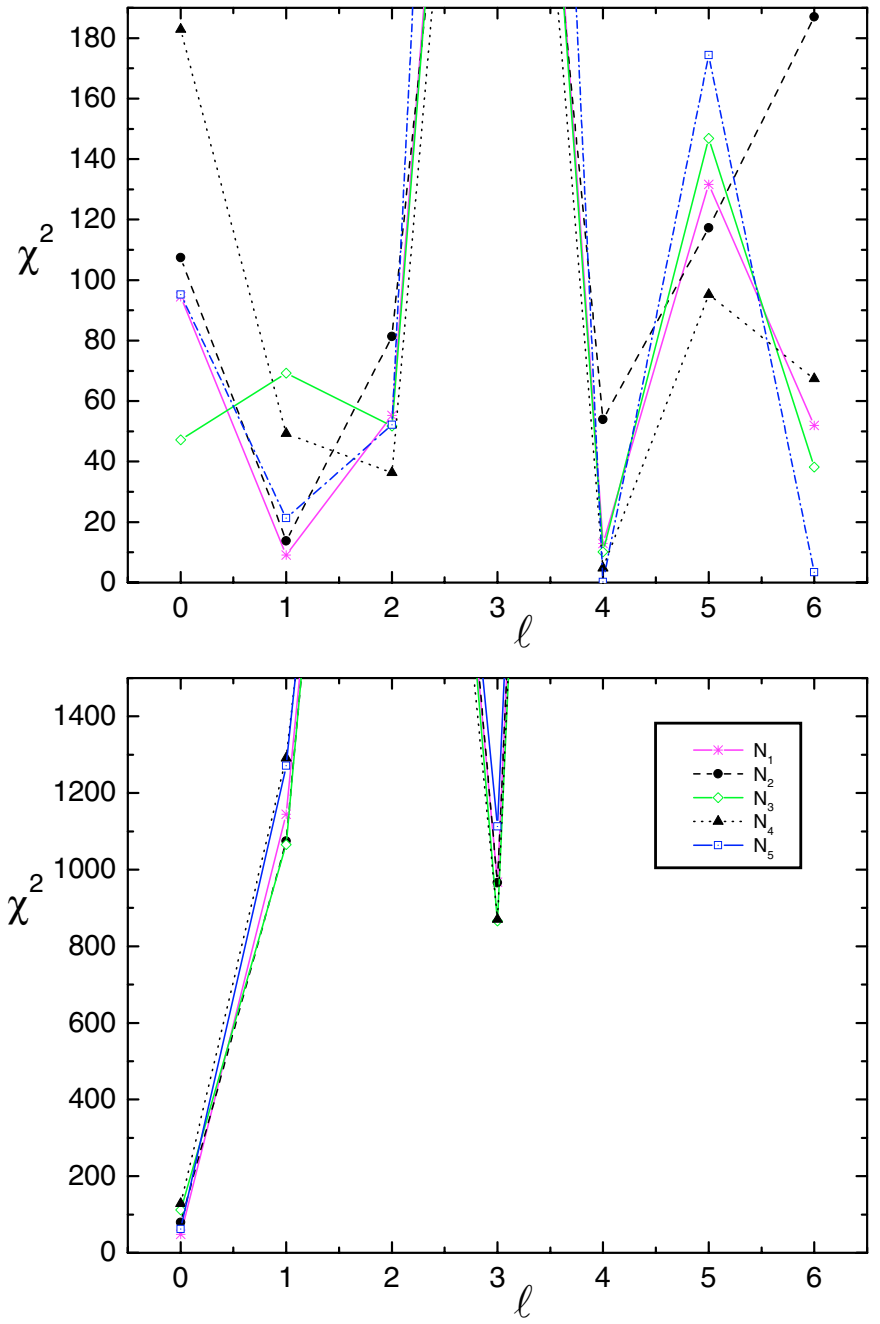

Fig. 8. The $\chi^{2}$ per degree of freedom as a function of $\ell$ obtained from the fit of photometric amplitudes and phases without radial velocity data (upper panel) and with radial velocity data (lower panel), for the dominant frequency, $v_{1}$. The five lines correspond to models from Table 1 which are consistent with the observations.

The next questionable part of our method concerns usage of equilibrium atmosphere models for evaluation of the flux derivatives in Eq. (1). Is the assumption that $\delta \mathcal{F}$ and $\delta r$ are constant within the atmosphere justified? Are the tabular data sufficiently accurate? After the discussion of Cugier et al. (1994), we are confident about a positive answer to the first question. We are less confident in the case of the second question but it seems unlikely that this is the cause of large $\chi^{2}$ because in the case of $\delta$ Ceti the $\chi^{2}$ minima were about 1 . We used atmospheric models from the same source and the stellar parameters of these two stars are very close.

Having rejected the inadequacy of the model, we turn to the error estimate. Let us first note that the main contribution to $\chi^{2}$ (above $90 \%$ ) comes from photometric data and that, in the case of the $v_{1}$ peak, we require a 7 times error increase of the photometric measurement to get $\chi \approx 1$. The errors given in Table 6 could be underestimated. The differences in amplitudes derived from various data sets exceeds the errors by a factor 5 . The problem was briefly discussed by 

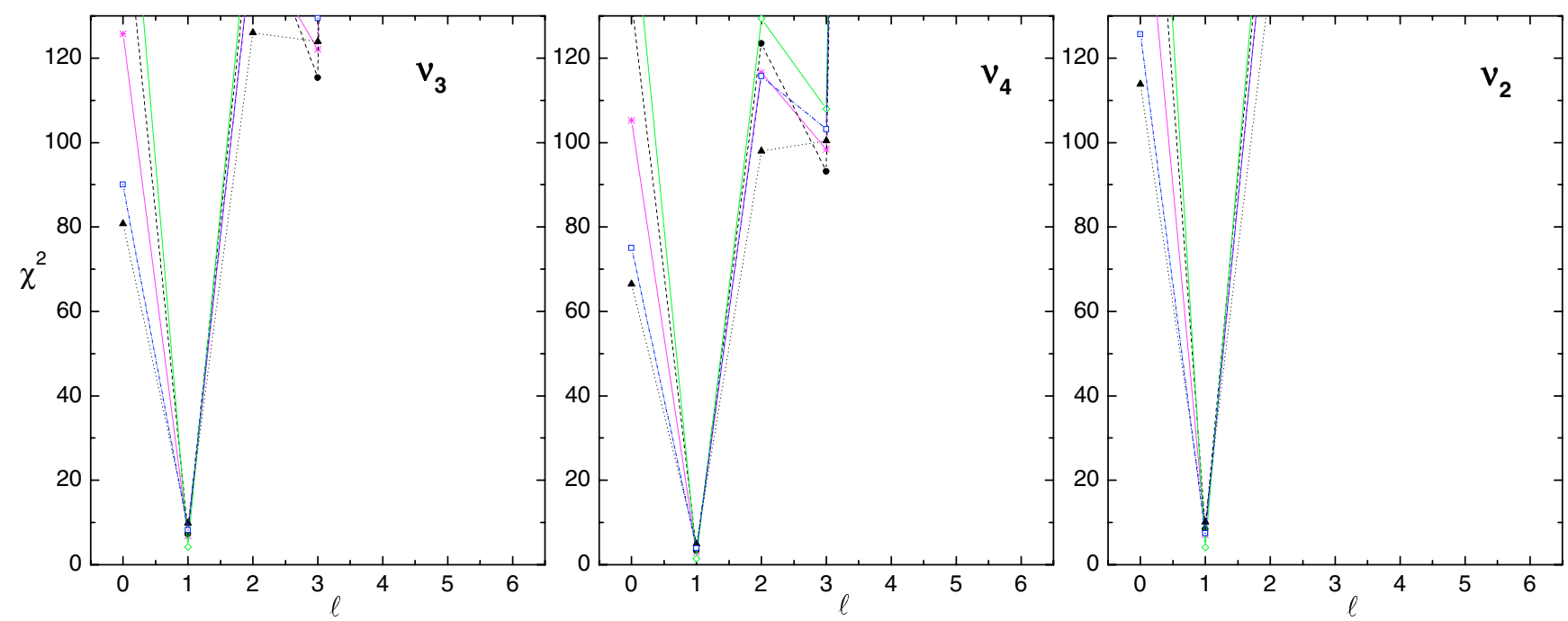

Fig. 9. The same as in the lower plot of Fig. 8 but for the close frequency triplet, $v_{3}, v_{4}, v_{2}(\ell=1, m=-1,0,+1)$.

Table 6. Values of $\tilde{\varepsilon}, f$ and $\chi^{2}$ for models of $v$ Eridani.

\begin{tabular}{|c|c|c|c|c|c|}
\hline & $\tilde{\varepsilon}_{\mathrm{R}}$ & $\tilde{\varepsilon}_{I}$ & $f_{\mathrm{R}}$ & $f_{\mathrm{I}}$ & $\chi^{2}$ \\
\hline \multicolumn{6}{|c|}{$v_{1}=5.76326 \mathrm{c} / \mathrm{d}, \ell=0$} \\
\hline N1 & $-0.0125(6)$ & $-0.0118(6)$ & $-9.2(4)$ & $0.8(4)$ & 48.4 \\
\hline $\mathrm{N} 2$ & $-0.0133(8)$ & $-0.0126(8)$ & $-8.8(5)$ & $0.7(5)$ & 79.6 \\
\hline N3 & $-0.0152(11)$ & $-0.0143(11)$ & $-8.1(5)$ & $0.6(5)$ & 112.0 \\
\hline N4 & $-0.0104(8)$ & $-0.0099(8)$ & $-10.6(7)$ & $0.9(7)$ & 128.4 \\
\hline N5 & $-0.0119(7)$ & $-0.0113(7)$ & $-9.6(4)$ & $0.8(4)$ & 61.8 \\
\hline \multicolumn{6}{|c|}{$v_{3}=5.62009 \mathrm{c} / \mathrm{d}, \ell=1, m=-1$} \\
\hline N1 & $0.0010(3)$ & $-0.0080(3)$ & $-9.0(3)$ & $0.1(3)$ & 6.81 \\
\hline $\mathrm{N} 2$ & $0.0011(3)$ & $-0.0085(3)$ & $-8.4(3)$ & $0.1(3)$ & 7.27 \\
\hline N3 & $0.0012(3)$ & $-0.0094(3)$ & $-7.5(2)$ & $0.1(2)$ & 4.15 \\
\hline N4 & $0.0009(3)$ & $-0.0067(3)$ & $-10.9(5)$ & $0.1(5)$ & 9.84 \\
\hline N5 & $0.0010(3)$ & $-0.0075(3)$ & $-9.6(4)$ & $0.1(4)$ & 8.22 \\
\hline \multicolumn{6}{|c|}{$v_{4}=5.63715 \mathrm{c} / \mathrm{d}, \ell=1, m=0$} \\
\hline N1 & $0.0008(2)$ & $0.0074(2)$ & $-9.0(2)$ & $0.5(2)$ & 3.02 \\
\hline $\mathrm{N} 2$ & $0.0009(2)$ & $0.0079(2)$ & $-8.4(2)$ & $0.5(2)$ & 3.40 \\
\hline N3 & $0.0010(1)$ & $0.0088(1)$ & $-7.5(1)$ & $0.5(1)$ & 1.46 \\
\hline N4 & $0.0007(2)$ & $0.0063(2)$ & $-10.9(3)$ & $0.6(3)$ & 4.95 \\
\hline N5 & $0.0008(2)$ & $0.0070(2)$ & $-9.6(3)$ & $0.6(3)$ & 3.93 \\
\hline \multicolumn{6}{|c|}{$v_{2}=5.65391 \mathrm{c} / \mathrm{d}, \ell=1, m=1$} \\
\hline N1 & $0.0036(3)$ & $-0.0080(3)$ & $-9.1(3)$ & $0.1(3)$ & 7.07 \\
\hline $\mathrm{N} 2$ & $0.0038(3)$ & $-0.0086(3)$ & $-8.5(3)$ & $0.1(3)$ & 8.34 \\
\hline N3 & $0.0042(3)$ & $-0.0094(3)$ & $-7.5(2)$ & $0.1(2)$ & 4.06 \\
\hline N4 & $0.0030(3)$ & $-0.0068(3)$ & $-11.0(5)$ & $0.1(5)$ & 10.07 \\
\hline N5 & $0.003493)$ & $-0.0076(3)$ & $-9.7(3)$ & $0.1(3)$ & 7.49 \\
\hline
\end{tabular}

Jerzykiewicz et al. (2005) who suggest only a factor of 2 error enhancement. A more sophisticated approach to the error analysis is needed but it is beyond the scope of the present work. We proceed further relying on determined $f$ values, being encouraged by the their consistency and reasonably low errors as well by the clear $\ell$ discrimination.

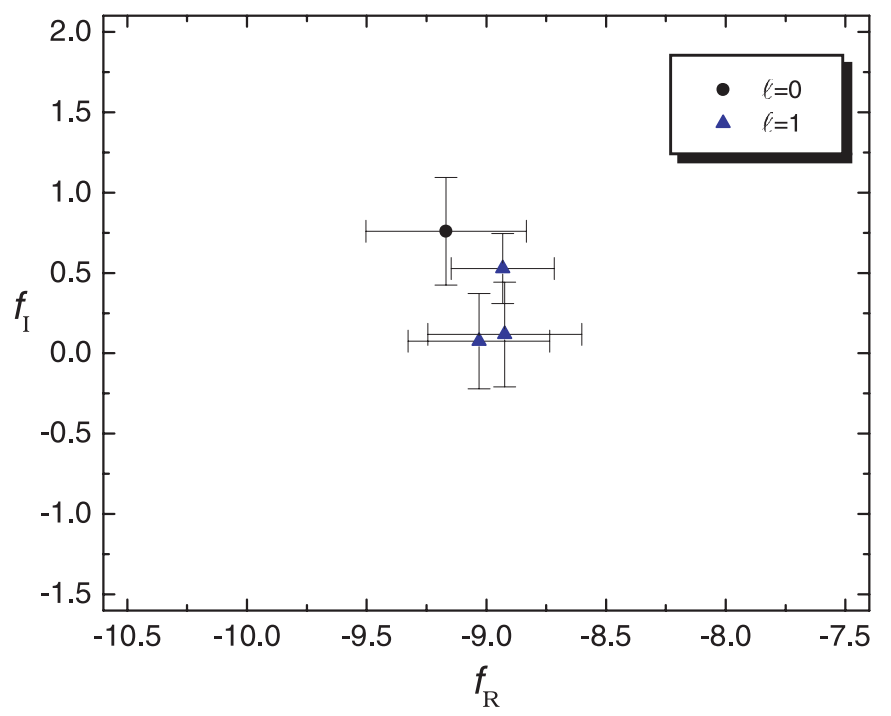

Fig. 10. Empirical $f$ values for $v_{1},(\ell=0)$, and the close triplet $v_{2}, v_{3}, v_{4},(\ell=1)$ for $\log T_{\text {eff }}=4.355$ and $\log L / L_{\odot}=3.969$.

In our comparison of empirical and theoretical values of $f$, we will consider only the $\ell=0$ mode because the errors are the lowest in that case. It is also safer than using the average from the four modes because there may be a noticeable effect of difference in frequencies. Values of $f$ for modes at more distant frequencies would be much more useful for estimating the $f(v)$ dependence, which is of interest. Unfortunately, we did not succeed in a meaningful determination of $f$ for any high frequency mode in $v$ Eri.

Comparison of the two panels of Fig. 11 shows clearly that the fundamental mode identification is preferred. The same identification was obtained by Pamyatnykh et al. (2004) and Ausseloos et al. (2004) who constructed seismic models $v$ Eri based on a simultaneous fit of the radial and dipole mode frequencies measured in the star.

As in the case of the $\delta$ Cet model, we find that opacity significantly influences the theoretical $f$ but, regardless of the source of the opacity data, the fundamental radial mode is the 

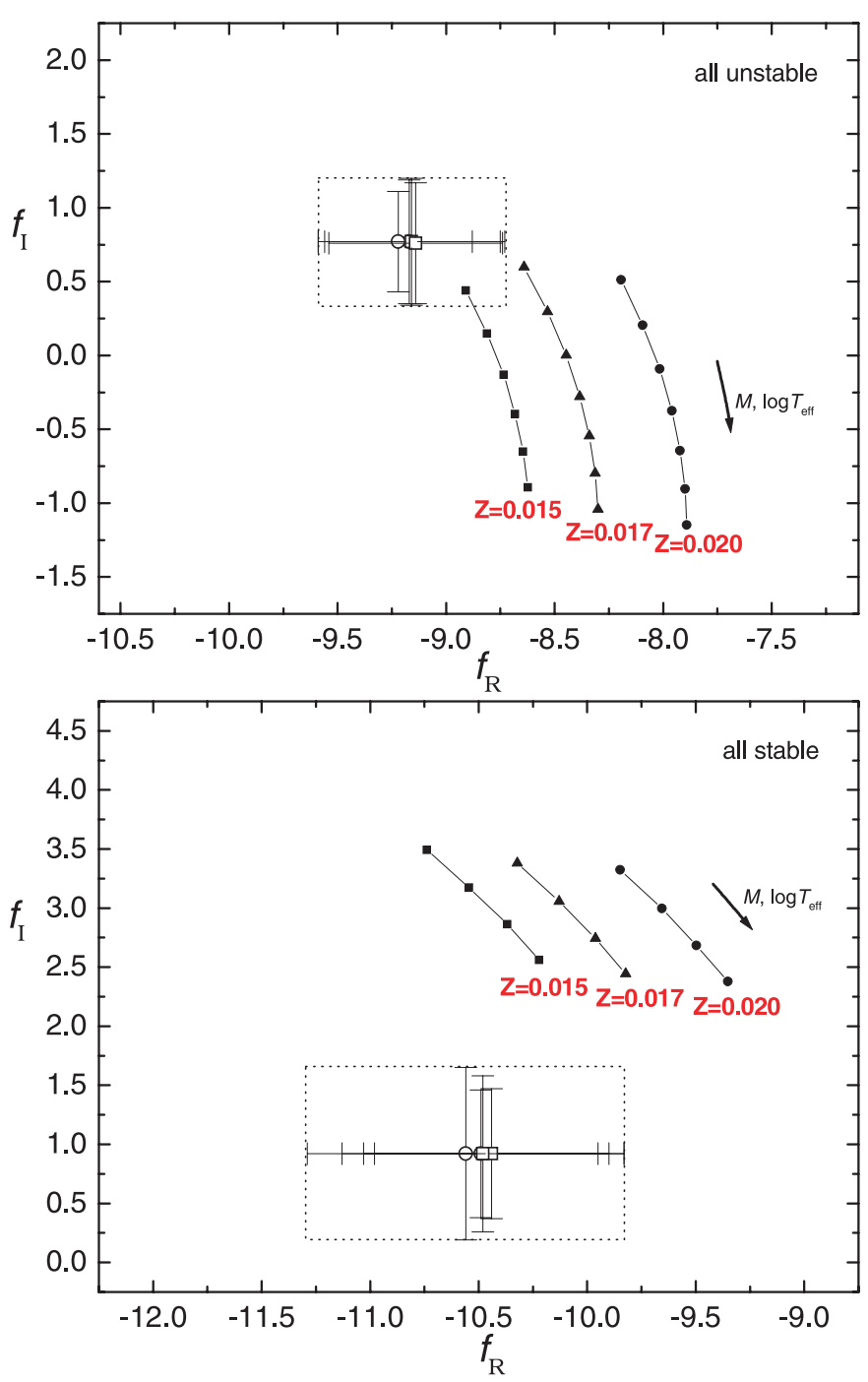

Fig. 11. Theoretical and empirical $f$ for the radial mode $\left(v_{1}\right)$ assuming various stellar parameters within the error box. The upper panel assumes fundamental and the lower one the first overtone mode identification. The empirical values are taken from Table 6 . Theoretical $f$ were obtained adopting the three indicated values of the metallicity parameter, $Z$, and the OPAL opacities. In the upper panel, the masses at the top are 9.0, 9.0, 9.2 $M_{\odot}$ for $Z=0.015,0.017,0.020$, respectively, whereas in the lower panel the masses at the top are 9.6, $9.8,10.0 M_{\odot}$ for $Z=0.015,0.017,0.020$, respectively. The step in mass is $0.2 M_{\odot}$.

only acceptable identification. Comparing Fig. 12 with the upper panel of Fig. 11, we see somewhat better agreement between the empirical and theoretical values when the OP opacities are used. However, the inference on the star parameter is significantly different. For instance, using OP data we get the mass higher by about $0.4 M_{\odot}, Z$ lower by about 0.0015 , and $\log T_{\text {eff }}$ higher by 0.01 . The above conclusions are based on stellar models constrained only by mean parameters from photometry and the radial mode frequency, $v_{1}$. We will see that our new seismic models lead us to somewhat different results.

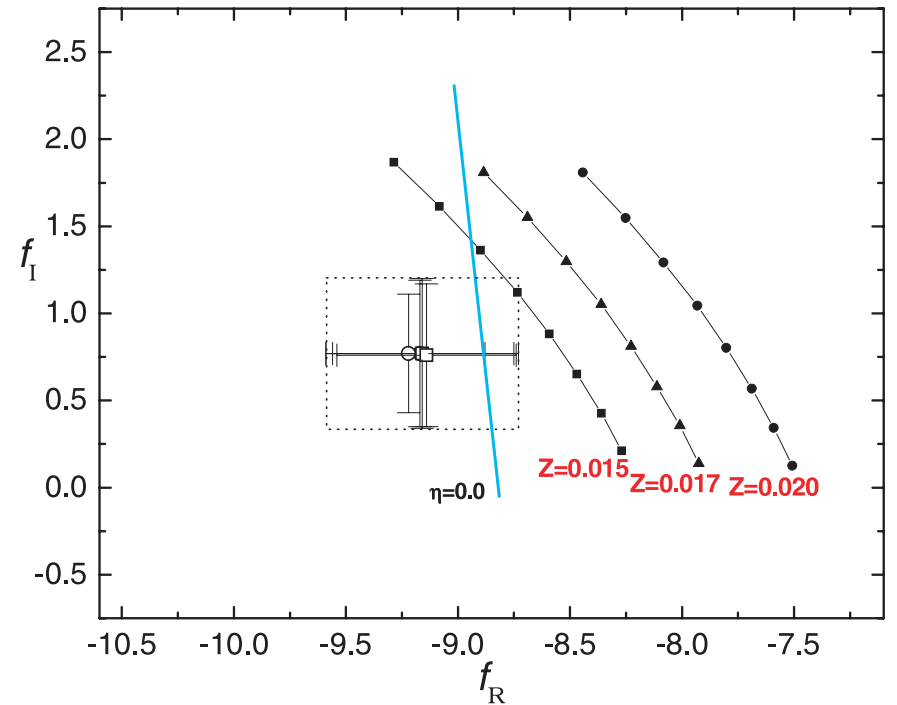

Fig. 12. The same as in the upper panel of Fig. 11 but with the theoretical $f$ values calculated with the OP opacities. Models lying to the right of the line marked with $\eta=0.0$ are unstable. The masses at the top are $8.8,9.0,9.2 M_{\odot}$ for $Z=0.015,0.017,0.020$, respectively. The step in mass is $0.2 M_{\odot}$.

\subsection{New seismic models}

We constructed seismic models using both OPAL and OP data. Like Pamyatnykh et al. (2004), the construction uses frequencies of the $\ell=0, p_{1}, \ell=1, g_{1}$ and $\ell=1, p_{1}$ modes. Models calculated with OPAL are slightly different than those of Pamyatnykh et al. (2004) due to a higher accuracy demand on the frequency fit. The model parameters and the $f$ values for $\ell=0, p_{1}$ are listed in Table 7. For each of the opacities, we provide data for the models obtained with two values of the overshooting parameter, $\alpha_{\mathrm{ov}}=0$ and 0.1 . In the seismic models, the influence of $\alpha_{\text {ov }}$ on $f$ enters indirectly through $T_{\text {eff }}$ and $Z$. We may see that the parameters of the models are significantly affected by the choice of the opacity data. Models calculated with the OP data are cooler by about $0.015 \mathrm{dex}$. The model calculated with $\alpha_{\mathrm{ov}}=0.1$ is in fact somewhat outside the allowed

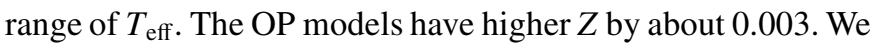
see that largest differences between the OPAL and OP models are in the imaginary part of $f$. Indeed the value of $f$ is a very sensitive probe of the stellar opacities.

How the $f$ values from the four seismic models compare with observations is shown in Fig. 13. The corresponding empirical $f$ are also given in Table 8 . For none of the opacity data is the agreement fully satisfactory. The truth seems to be somewhere in between, if a moderate overshooting is allowed. Relying only on OPAL opacities, we may reach the agreement by increasing $\alpha_{\mathrm{ov}}$ to about 0.2 . However, a further increase would move the model off the instability range.

\section{Conclusions}

Using amplitudes and phases from simultaneous photometric and spectroscopic observations of two $\beta$ Cep stars, we derived the spherical harmonic degree, $\ell$, of excited modes and the complex parameter, $f$, which may be directly compared with 
Table 7. Seismic models of $v$ Eri calculated with the OPAL and OP opacities. The $f$ values are given for $\ell=0, p_{1}$.

\begin{tabular}{cccccccc}
\hline \hline & $M / M_{\odot}$ & $\alpha_{\mathrm{ov}}$ & $Z$ & $\log T_{\text {eff }}$ & $\log L$ & $f_{\mathrm{R}}$ & $f_{I}$ \\
\hline \multicolumn{7}{c}{ OPAL } \\
\hline S1 & 9.808 & 0.0 & 0.0155 & 4.3530 & 3.959 & -8.56 & -0.63 \\
S2 & 9.230 & 0.1 & 0.0145 & 4.3424 & 3.903 & -8.82 & -0.07 \\
\hline \multicolumn{7}{c}{ OP } \\
\hline S3 & 9.590 & 0.0 & 0.0185 & 4.3396 & 3.902 & -8.22 & 1.20 \\
S4 & 9.020 & 0.1 & 0.0175 & 4.3284 & 3.843 & -8.70 & 1.70 \\
\hline
\end{tabular}

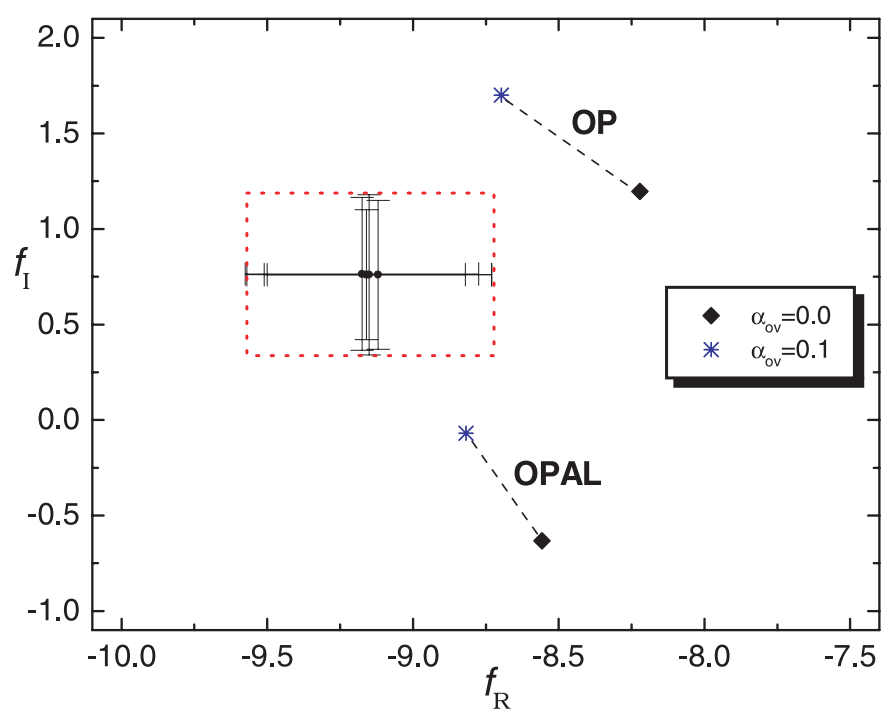

Fig. 13. The $f$ values for fundamental radial mode of the seismic models constructed using opacity data from two sources (OPAL and OP) and using two values of the overshooting parameter $\left(\alpha_{\mathrm{ov}}=0.0\right.$ and 0.1 ). The empirical $f$ values were obtained assuming atmospheric parameters corresponding to the seismic models.

the nonadiabatic theory of stellar oscillations. The $f$ parameter determines bolometric flux amplitude at specified surface displacement and it constitutes a new seismic probe of stellar outer layers.

One of the stars is a monoperiodic pulsator $\delta$ Cet, the other is a multiperiodic pulsator $v$ Eri. In the latter star, we analyzed data on four dominant peaks. We found that unique determination of mode degree requires both photometric and spectroscopic data. In our analysis we relied on the use of static atmospheric models of Kurucz (2004).

Empirical values of $f$ were compared with the theoretical values for relevant stellar models. The models employed the OPAL (Iglesias \& Rogers 1996) and the OP tabular data (Seaton \& Badnell 2004; Badnell et al. 2005; Seaton 2005). The evolutionary tracks are not much affected by the choice of the data but significant differences are seen in the resulting $f$.

We confirmed the $\ell=0$ identification for the only mode detected in $\delta$ Cet but, unlike our predecessors, we think that the mode is more likely fundamental than the first overtone. The reason is that the latter mode is stable over the allowed range of mean star parameters. If indeed the mode is fundamental
Table 8. Values of $\tilde{\varepsilon}, f$ and $\chi^{2}$ for seismic models of $v$ Eridani for the dominant frequency, $v_{1}$.

\begin{tabular}{lccccc}
\hline \hline & $\tilde{\varepsilon}_{\mathrm{R}}$ & $\tilde{\varepsilon}_{\mathrm{I}}$ & $f_{\mathrm{R}}$ & $f_{\mathrm{I}}$ & $\chi^{2}$ \\
\hline \multicolumn{5}{c}{$v_{1}=5.76326 \mathrm{c} / \mathrm{d}, \ell=0$} \\
\hline S1 & $-0.0126(6)$ & $-0.0120(6)$ & $-9.2(3)$ & $0.8(3)$ & 40.2 \\
S2 & $-0.0128(7)$ & $-0.0121(7)$ & $-9.1(4)$ & $0.8(4)$ & 55.8 \\
S3 & $-0.0126(7)$ & $-0.0120(7)$ & $-9.2(4)$ & $0.8(4)$ & 56.4 \\
S4 & $-0.0127(7)$ & $-0.0121(7)$ & $-9.2(4)$ & $0.8(4)$ & 61.9 \\
\hline
\end{tabular}

then approximate consistency of the $f$ values is possible only when the OP opacities are used.

Our identification of the four dominant peaks in the $v$ Eri oscillation spectrum is the same as that of Pamyatnykh et al. (2004) and Ausseloos et al. (2004). Consistency between the theoretical and empirical values of $f$ was achieved both with OPAL and OP data, but at significantly different stellar parameters.

For none of the two opacity data did we achieve full agreement between empirical $f$ and those derived from seismic models fitting three dominant centroid frequencies in the oscillation spectrum. As one can see, a perfect fit would be possible at some intermediate opacities assuming a small overshooting parameter, $\alpha_{\mathrm{ov}}$.

There are things to improve in our models of $\beta$ Cep stars. As Pamyatnykh et al. (2004) pointed out, the standard models do not account for excitation of modes at very low and very high frequencies in $v$ Eri. We also cannot be fully satisfied with our fit of empirical and theoretical $f$. These two problems may be related. The solution to the first problem proposed by Pamyatnykh et al. (2004) was a significant enhancement of the iron abundance in the driving zone caused by the selective radiation pressure. Undoubtedly, this will be reflected in the $f$ values. We plan to investigate the effect when we have physically justified models of the enhancement.

Ideal seismic stellar models should account, within the errors, not only for all measured frequencies but also for the $f$ values. Such models will provide an ultimate test of our knowledge of the stellar structure from the atmosphere to the deep interior. We are not at this point yet. We believe that the most important result of the present work is that our new seismic tool provides a stringent probe of stellar opacities.

Acknowledgements. We are grateful to Mike Jerzykiewicz for kindly providing data on photometric amplitudes and phases as well as for illuminating discussion about possible sources of errors. We thank Mike Seaton for detailed discussion of how to use his new opacity data, and Conny Aerts for making spectra of $\delta$ Cet and $v$ Eri accessible. We also thank the anonymous referee for stimulating comments. J.D.D. thanks the Foundation for Polish Science for supporting her stay in the Copernicus Astronomical Center. The work was partially supported by MNiI grant No. 1 P03D 02128.

\section{References}

Ausseloos, M., Scuflaire, R., Thoul, A., \& Aerts, C. 2004, MNRAS, 355,352

Aerts, C., de Cat, P., Handler, G., et al. 2004, MNRAS, 347, 463 
Aerts, C., de Pauw, M., \& Waelkens, C. 1992, A\&A, 266, 294

Badnell, N. R., Bautista, M. A., Butler, K., et al. 2005, MNRAS, 360, 458

Claret, A. 2000, A\&A, 363, 1081

Cugier, H., Dziembowski, W. A., \& Pamyatnykh, A. A. 1994, A\&A, 291, 143

Cugier, H., \& Nowak, D. 1997, A\&A, 326, 620

Daszyńska-Daszkiewicz, J., Dziembowski, W. A., Pamyatnykh, A. A., \& Goupil, M.-J. 2002, A\&A, 392, 151

Daszyńska-Daszkiewicz, J., Dziembowski, W. A., \& Pamyatnykh, A. A. 2003, A\&A, 407, 999

Daszyńska-Daszkiewicz, J., Dziembowski, W. A., Pamyatnykh, A. A., Breger, M., \& Zima, W. 2004, Proc. IAU Symp. 224, ed. J. Zverko, W. W. Weiss, J. Ziznovsky, \& S. J. Adelman, 853

Grevesse, N., \& Noels, A. 1993, in Origin and Evolution of the Elements, ed. N. Pratzo, E. Vangioni-Flam, \& M. Casse (Cambridge Univ. Press), 15

Handler, G., Shobbrook, R. R., Jerzykiewicz, M., et al. 2004, MNRAS, 347, 454
Iglesias, C. A., \& Rogers, F. J. 1996, ApJ, 464, 943

Jerzykiewicz, M., Handler, G., Shobbrook, R. R., et al. 2005, MNRAS, 360, 619

Kołaczkowski, Z., Pigulski, A., Soszyński, I., et al. 2004, in Variable Stars in the Local Group, Proc. IAU Coll., 193, ed. D. W. Kurtz, \& K. R. Pollard, ASP Conf. Ser., 310, 225

Kubiak, M., \& Seggewiss, W. 1990, Acta Astron., 40, 85

Kurucz, R. L. 2004, http: //kurucz .harvard.edu

Lutz, T. E., \& Kelker, D. H. 1973, PASP, 85, 573

Pamyatnykh, A. A., Handler, G., \& Dziembowski, W. A. 2004, MNRAS, 350, 1022

de Ridder, J., Telting, J. H., Balona, L. A., et al. 2004, MNRAS, 351, 324

Seaton, M. J. 2005, MNRAS, in press [arXiv: astro-ph/0411010]

Seaton, M. J., \& Badnell, N. R. 2004, MNRAS, 354, 457

Seaton, M. J., Yu Yan, Mihalas, D., \& Pradhan, A. K. 1994, MNRAS, 266, 805

Stankov, A., \& Handler, G. 2005, ApJS, 158, 193 